\title{
Empirical assessment of state-and-transition models with a long-term vegetation record from the Sonoran Desert
}

\author{
Sumanta Bagchi,${ }^{1,5}$ David D. Briske,,${ }^{1}$ X. B. Wu,,${ }^{1}$ Mitchel P. McClaran, ${ }^{2}$ Brandon T. Bestelmeyer,${ }^{3}$ \\ and Maria E. Fernández-Giménez ${ }^{4}$ \\ ${ }^{1}$ Department of Ecosystem Science and Management, Texas A\&M University, 2138 TAMU, College Station, Texas 77843 USA \\ ${ }^{2}$ School of Natural Resources and the Environment, University of Arizona, 325 Biosciences East, Tucson, Arizona 85721 USA \\ ${ }^{3}$ USDA-ARS, Jornada Experimental Range and Jornada Basin LTER, MSC 3JER, Box 30003, New Mexico State University, \\ Las Cruces, New Mexico 88003 USA \\ ${ }^{4}$ College of Natural Resources, Colorado State University, Fort Collins, Colorado 80523 USA
}

\begin{abstract}
Resilience-based frameworks, including state-and-transition models (STM), are being increasingly called upon to inform policy and guide ecosystem management, particularly in rangelands. Yet, multiple challenges impede their effective implementation: (1) paucity of empirical tests of resilience concepts, such as alternative states and thresholds, and (2) heavy reliance on expert models, which are seldom tested against empirical data. We developed an analytical protocol to identify unique plant communities and their transitions, and applied it to a long-term vegetation record from the Sonoran Desert (1953-2009). We assessed whether empirical trends were consistent with resilience concepts, and evaluated how they may inform the construction and interpretation of expert STMs. Seven statistically distinct plant communities were identified based on the cover of 22 plant species in 68 permanent transects. We recorded 253 instances of community transitions, associated with changes in species composition between successive samplings. Expectedly, transitions were more frequent among proximate communities with similar species pools than among distant communities. But unexpectedly, communities and transitions were not strongly constrained by soil type and topography. Only 18 transitions featured disproportionately large compositional turnover (species dissimilarity ranged between 0.54 and 0.68 ), and these were closely associated with communities that were dominated by the common shrub (burroweed, Haplopappus tenuisecta); indicating that only some, and not all, communities may be prone to large compositional change. Temporal dynamics in individual transects illustrated four general trajectories: stability, nondirectional drift, reversibility, and directional shifts that were not reversed even after 2-3 decades. The frequency of transitions and the accompanying species dissimilarity were both positively correlated with fluctuation in precipitation, indicating that climatic drivers require more attention in STMs. Many features of the expert models, including the number of communities and participant species, were consistent with empirical trends, but expert models underrepresented recent increases in cacti while overemphasizing the introduced Lehmann's lovegrass (Eragrostis lehmanniana). Quantification of communities and transitions within long-term vegetation records presents several quantitative metrics such as transition frequency, magnitude of accompanying compositional change, presence of unidirectional trajectories, and lack of reversibility within various timescales, which can clarify resilience concepts and inform the construction and interpretation of STMs.
\end{abstract}

Key words: alternative stable states; cluster analysis; detrended correspondence analysis; dynamic regimes; nonequilibrium theory; rangeland management; regime shifts; thresholds.

\section{INTRODUCTION}

Resilience-based ecological frameworks, founded upon the existence of multiple attractors and alternative states, are being increasingly called upon to guide ecosystem management and inform natural resource policy (Cumming et al. 2005, King and Hobbs 2006, Gillson and Hoffman 2007, Briske et al. 2008).

Manuscript received 18 April 2011; revised 20 September 2011; accepted 21 September 2011. Corresponding Editor (ad hoc): N. P. Hanan.

${ }^{5}$ E-mail: sbagchi@ag.tamu.edu
Resilience represents the ability of ecosystems to absorb external forcing, either natural or anthropogenic, without major alterations to structure and function (Holling 1973). The resilience-based approach represents a substantial departure from the Clementsian successional paradigm (Dyksterhuis 1949), as it posits that ecosystems may have multiple attractors, or alternative states, rather than a single equilibrium (e.g., a climax plant community). This approach recognizes that system resilience can be exceeded to yield alternative states that are associated with threshold conditions and underlying feedback mechanisms (Gunderson 2000, Scheffer et al. 
2001). For example, communities of macrophytes and phytoplankton may represent alternative states in shallow lakes and estuaries (Scheffer et al. 1993), while grasslands and woodlands may indicate alternative states in rangeland ecosystems (Archer et al. 1988).

Resilience-based management is especially relevant to arid and semiarid rangelands (Gillson and Hoffman 2007), because they are particularly vulnerable to threshold conditions that may compromise the provisioning of ecosystem services, and adversely affect human well-being (Reynolds et al. 2007, Scheffer 2009). State-and-transition models (STM) have been developed to incorporate "resilience thinking" into rangeland management (Westoby et al. 1989, Briske et al. 2008). Conceptually, STMs are organized as a collection of potential alternative states, corresponding to multiple attractors, separated by thresholds. Each state may consist of one or more community phases that can undergo reversible within-state dynamics, often without compromising ecosystem function (Westoby et al. 1989, Stringham et al. 2003, Bestelmeyer et al. 2004). Transitions among alternative community phases, as well as between states, are variously linked to the interactive effects of human interventions, autogenic succession, and climatic drivers (Archer et al. 1988, Watson et al. 1996, Briske et al. 2005).

In spite of the broad relevance of resilience concepts, and a recognized need for resilience-based management, several major challenges continue to impede their implementation. The primary challenges include: (1) a paucity of empirical tests of resilience concepts such as alternative states, transitions, and thresholds (Walker and Wilson 2002, Briske et al. 2003); and (2) uncertainties surrounding empirical attributes of thresholds and corresponding early warning signals (Scheffer et al. 2009, Hastings and Wysham 2010). As a consequence, rangeland managers rely heavily, and often exclusively, on models developed with expert opinion and experiential knowledge (Knapp and Fernandez-Gimenez 2009, Knapp et al. 2011). Expert models are of great value for communicating information to aid land management decisions, yet considerable ambiguity persists in the interpretation of resilience concepts that hinders effective communication between researchers and practitioners (Knapp et al. 2011). In addition, broad acceptance and application of the STM framework over the past 25 years has established a need for quantitative approaches to address the drivers and feedback mechanisms responsible for transitions between alternative states (Walker and Wilson 2002, Briske et al. 2003).

Here we developed an analytical protocol to inform the construction and interpretation of STMs with empirical patterns derived from a long-term vegetation record. We evaluated long-term (1953-2009) vegetation dynamics from the Santa Rita Experimental Range (SRER) that encompasses nearly $200 \mathrm{~km}^{2}$ of the Sonoran Desert in Arizona, USA (McClaran et al. 2003). This vegetation record consists of changes in plant species' cover that was measured 26 times from replicate permanent transects (interval $2.24 \pm 0.3$ years $[$ mean $\pm \mathrm{SE}]$ ). Vegetation dynamics on the SRER have not been greatly influenced by management interventions (e.g., brush control, prescribed fire, and seeding; McClaran et al. 2003), which provides an opportunity to assess the role of autogenic changes in relation to climatic drivers and topo-edaphic conditions. Specifically, we (1) developed a protocol for analyzing temporal trends in vegetation composition, using cluster analysis and ordination, that can identify plant communities and transition pathways; (2) investigated the relationship among community transitions, precipitation patterns, and topo-edaphic conditions; and (3) investigated the degree of correspondence between expert STMs and the patterns observed in the empirical record.

\section{Materials And Methods}

\section{Study area}

Topography of SRER consists of hills, basins, fan terraces, and floodplains that cover an elevation range of 880-1400 m. Annual precipitation ranges between 275 and $450 \mathrm{~mm}$ (mean $362 \mathrm{~mm}$; 1922-2009), with over $60 \%$ occurring during the summer months. Native perennial species include $\mathrm{C}_{4}$ grasses (e.g., black grama Bouteloua eriopoda, sprucetop grama $B$. chrondrosoides, rothrock grama B. rothrockii, bush muhly Muhlenbergia porteri, cottontop Digitaria californica), forbs, and shrubs (e.g., burroweed Haplopappus tenuisecta, shortleaf baccharis Baccharis brachyphylla, fairy duster Calliandra eriophylla, velvetpod mimosa Mimosa dysocarpa, dwarf zinnia Zinnia pumila), cacti (e.g., prickly pear Opuntia engelmannii, chainfruit cholla $O$. fulgida), and woody species such as velvet mesquite (Prosopis velutina). Lehmann's lovegrass (Eragrostis lehmanniana), a $\mathrm{C}_{4}$ perennial, was introduced in the 1930s (see Plate 1).

\section{Data set}

Vegetation plots and transects were established at various times on the SRER, with the oldest dating to 1903 (McClaran et al. 2003). In order to use the largest possible set of repeated measurements with a consistent sampling protocol, data were analyzed from a total of 68 transects that did not experience direct vegetation manipulations (seeding or brush removal, or fire; Fig. 1). Of these, data were available from 1953 onwards in 10 transects, from 1957 onwards in 48 transects, and 1959 onwards for the remaining 10 transects. These transects were distributed throughout SRER, and encompassed various topo-edaphic locations (Fig. 1). According to ecological site descriptions (ESD) developed by the Natural Resource Conservation Service (NRCS) sandy loam upland ( $n=22$ transects), sandy loam deep $(n=20)$, and loamy upland $(n=12)$ represented the major topographical and edaphic conditions (Breckenfield and Robinett 2003). Six other ESD categories (granitic hills, loamy slopes, sandy wash, sandy upland, limy upland, and clay loam upland) 


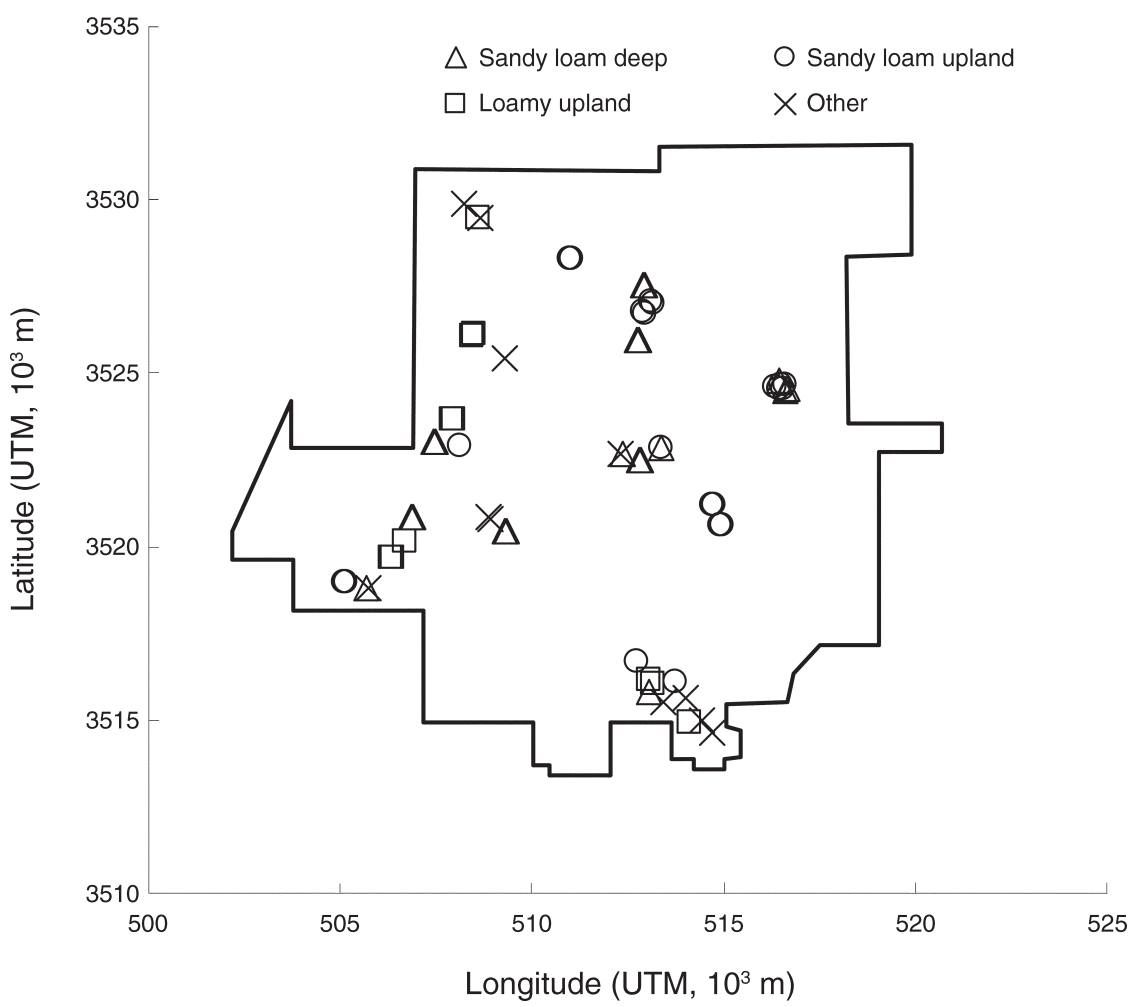

FIG. 1. Schematic map of the Santa Rita Experimental Range (SRER) in the Sonoran Desert in Arizona, USA, showing locations of the 68 transects used in our analysis. Different symbols correspond to topo-edaphic categories, or ecological site descriptions (ESD), developed by USDA Natural Resources Conservation Service (NRCS); the majority of these transects were in three ESD categories.

accounted for the remaining 14 transects with 1-3 transects represented in each ESD (Fig. 1). On every transect, absolute cover of perennial plants (canopy for shrubs and cacti, basal for perennial grasses) was measured at $3.05-\mathrm{cm}$ increments along a permanently marked 30.5-m line (further details in McClaran et al. 2003, McClaran and Angell 2007). Of the 67 plant species identified in this data set, only 22 species comprised $>2 \%$ cover in at least three transects, while the remaining species were relatively rare. We restricted our analysis to these 22 relatively common species in 68 transects that were sampled 26 times between 1953 and 2009. These data are available online from the University of Arizona. ${ }^{6}$

\section{Data analysis}

We adopted a heuristically simple analytical approach where vegetation communities were identified on the basis of plant species composition. The initial step was to identify the unique number of plant communities using cluster analysis. Several procedures exist to determine the number of clusters in a data set (Milligan and Cooper 1985), and we adopted a model-based approach to obtain a parsimonious estimate of the

${ }^{6}$ http://ag.arizona.edu/srer number clusters (Fraley and Raftery 2002). For models with varying numbers of clusters, model fit to data (and hence the most parsimonious estimate of the number of constituent communities) was based on Bayesian information criteria (BIC; Fraley and Raftery 2002). Once the number of clusters, or plant communities, was ascertained, we determined community membership of the individual samples using Ward's linkage over BrayCurtis dissimilarity index (Legendre and Legendre 1998). Subsequently, we used detrended correspondence analysis (DCA; Hill and Gauch 1980) to estimate relative separation between communities as distances over ordination space (Legendre and Legendre 1998). To verify that the identified communities were indeed distinguishable from each other, we used analysis of similarity (ANOSIM) to estimate within-group and between-group variation from pairwise compositional dissimilarity among the communities with Bray-Curtis index, and statistical significance was determined with 1000 Monte Carlo randomizations. Statistical significance in ANOSIM indicates that transects assigned to a community were relatively homogenous, and compositionally different from others transects assigned to alternative communities. To ascertain which species were most influential in demarcating community boundaries, we used similarity percentage (SIMPER) to 
examine the relative contribution of constituent species in each community.

Once individual transects were assigned to communities, we tracked them through time to identify compositional changes that resulted in reassignment to an alternative community between successive measurements. From this we assembled the historical dynamics of SRER vegetation by identifying the number of transitions among distinct plant communities (AllenDiaz and Bartolome 1998, Jackson and Bartolome 2002). We compared the empirical patterns with expert STMs available for the region (MLRA [major land use area] 40 and 41, NRCS; data available online). ${ }^{7}$ The time interval for the 26 sampling events was not uniform during the 56-year record, and this introduces the possibility that larger compositional dissimilarities may be associated with longer intervals. To assess the magnitude of this potential confounding influence, we evaluated the extent of covariation between compositional dissimilarity in transects and the time interval separating consecutive measurements.

Finally, we assessed whether communities and transitions were related to specific topo-edaphic conditions based on ESD, and with precipitation patterns based on the mean precipitation recorded at six locations in SRER during the 12 months preceding each sampling event. Since there were only 10 transects that were sampled between 1953 and 1956, we restricted this analysis to 1957-2009 (subset of $n=58$ transects).

We used packages Mclust and vegan in R 2.8.1 ( $\mathrm{R}$ Development Core Team 2008) for cluster analysis and ordination, and PRIMER 5 (Plymouth Marine Laboratory, Plymouth, UK) for SIMPER and ANOSIM.

\section{RESULTS}

\section{Plant communities and long-term vegetation dynamics}

The long-term vegetation record showed considerable dynamics in the cover of the 22 common plant species through time (Fig. 2). Across the entire SRER landscape, (1) burroweed has declined since the mid1980s, (2) mesquite and cacti increased during the same period, (3) Lehmann's lovegrass remained rare between 1950 and 1980, but increased since the mid-1980s, and (4) relative cover of native grasses and subordinate shrubs $(<10 \%$ cover $)$ fluctuated over time with no consistent or directional trend (Fig. 2). Cluster analyses identified seven parsimonious groups in the overall vegetation record; each of which can be viewed as a unique plant community. ANOSIM verified that species composition of these seven communities were significantly different from one another (Global $R=0.78, P<$ 0.001 , and $P<0.05$ for each pairwise comparison). These communities were: [A] fairy duster and mesquite co-dominated; [B] mesquite, burroweed, and prickly pear cacti mixed; [C] mesquite and burroweed; [D]

\footnotetext{
${ }^{7}$ http://esis.sc.egov.usda.gov
}

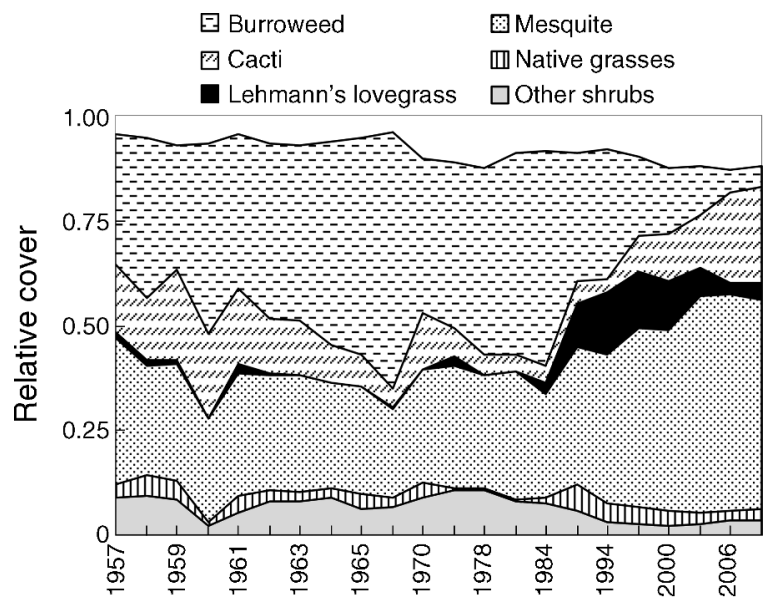

FIG. 2. Vegetation dynamics expressed as relative cover of key plant species and functional groups on the Santa Rita Experimental Range (SRER) over time.

burroweed and dwarf zinnia; [E] mesquite and Lehmann's lovegrass; [F] burroweed dominated; and [G] prickly pear cacti and burroweed (Fig. 3a). SIMPER revealed that among the pool of 22 relatively common species, only eight species were responsible for determining community differences, while the remaining species were less important (Figs. 2 and 3). Except for community [A], which was absent from the deep sandy loam ESD, the communities were distributed over multiple topo-edaphic categories (Fig. 3b). In general, topo-edaphic constraints on species composition were weak as all communities occurred over two to four ESD categories (Fig. 3b).

The relative separation of these communities in twodimensional ordination space (DCA; Fig. 4a) suggested that communities [A] and [D] were most distant from one another (average dissimilarity 0.92). In comparison, [B] and $[\mathrm{G}]$ were the most proximate (average dissimilarity 0.59). In general, all community pairs involving [B], [C], $[\mathrm{E}],[\mathrm{G}]$, and $[\mathrm{F}]$ were relatively close to each other, with their average dissimilarities ranging between 0.59 and 0.71 (Fig. 4a). The ordination scores of samples were only weakly related to their corresponding ESD. Except for one of the minor ESDs that occupied a distinct position in ordination space, i.e., granitic hills, there was considerable overlap among the others (Fig. 4b).

\section{Community transitions}

The seven distinct communities could result in a total of 42 potential transition pathways between them; $2\left(C_{2}^{7}\right)=7$ !/ $[2 !(7-2) !]=42 ; \mathrm{A} \rightarrow \mathrm{B}, \mathrm{B} \rightarrow \mathrm{A}, \mathrm{A} \rightarrow \mathrm{C}$, and so on. Thirtyseven of these transition pathways were recorded in the data set, amounting to a total of 253 instances that required reassignment of community membership (Table 1, Fig. 5). Communities with greater species similarity (Fig. 3a), as illustrated by DCA ordination (Fig. 4a), showed more frequent transitions, while more dissimilar 
a

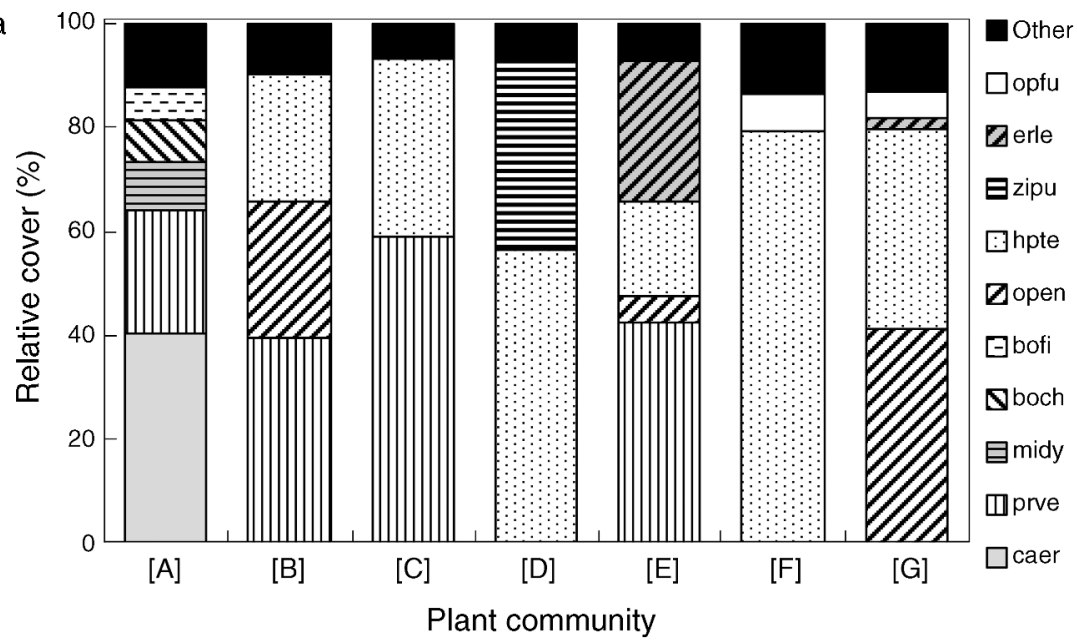

b

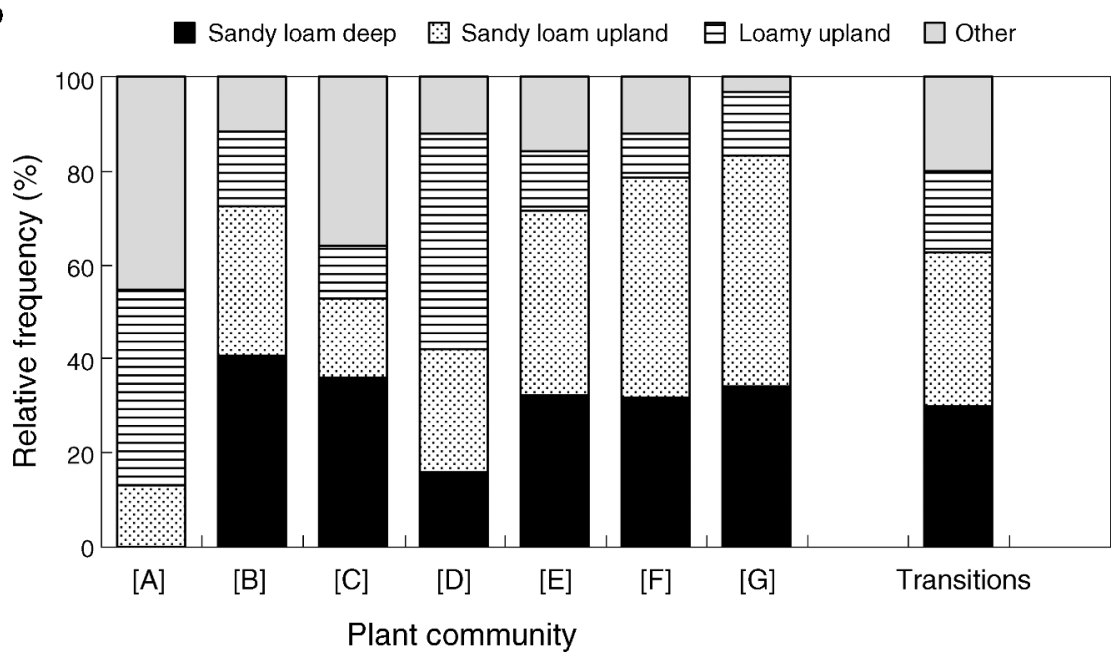

FIG. 3. (a) Mean relative abundance shown as percent cover of key plant species in seven unique plant communities, labeled as [A] through [G], on the Santa Rita Experimental Range (SRER) between 1953 and 2009. (b) Relative frequency of occurrence of the seven plant communities, and transitions between communities, across ecological site descriptions (ESD) developed by USDANRCS. Species abbreviations are: boch, Bouteloua chrondrosoides; bofi, Bouteloua filiformis; caer, Calliandra eriophyla; erle, Eragrostis lehmanniana; hpte, Haplopappus tenuisecta; midy, Mimosa dysocarpa; open, Opuntia engelmannii; opfu, Opuntia fulgida; prve, Prosopis velutina; and zipu, Zinnia pumila.

communities showed few or no transitions (Table 1, Fig. 5). In terms of relative susceptibility to change, [A] underwent the fewest transitions to an alternative community, while [B] transitioned most frequently (Table 1).

Compositional dissimilarity expressed during transitions $(0.31 \pm 0.004[$ mean $\pm \mathrm{SE}])$ was greater than dissimilarity in absence of transitions $(0.15 \pm 0.01$, $\left.F_{1,1403}=283.1, \quad P<0.001\right)$. The distribution of dissimilarities expressed during transitions was positively skewed (skewness $=1.43$, D'Agostino test $P<0.001$ ). Of the 253 transitions, none could be attributed to relatively small changes in composition such that the accompanying dissimilarity was two standard deviations below the mean. However, 18 transition events could be attributed to relatively large changes in composition since their accompanying dissimilarity was two standard deviations above the mean (between 0.54 and 0.68). Of these 18 , as many as 14 instances involved community $[F]$, while communities $[D]$ and $[A]$ were involved in the remaining four cases. In the corresponding transects, dynamics of burroweed, which dominated community $[F]$, showed large fluctuations over relatively short time intervals (Fig. 6). We detected a positive association between magnitude of compositional dissimilarity and sampling interval, but only a small fraction of the variation in compositional change was explained by time interval $\left(r^{2}=0.08, P=0.003\right)$.

\section{Comparison to expert models}

While the representative expert-based STM for SRER (Fig. 7) showed certain similarities with historical vegetation dynamics, there were also key differences. 

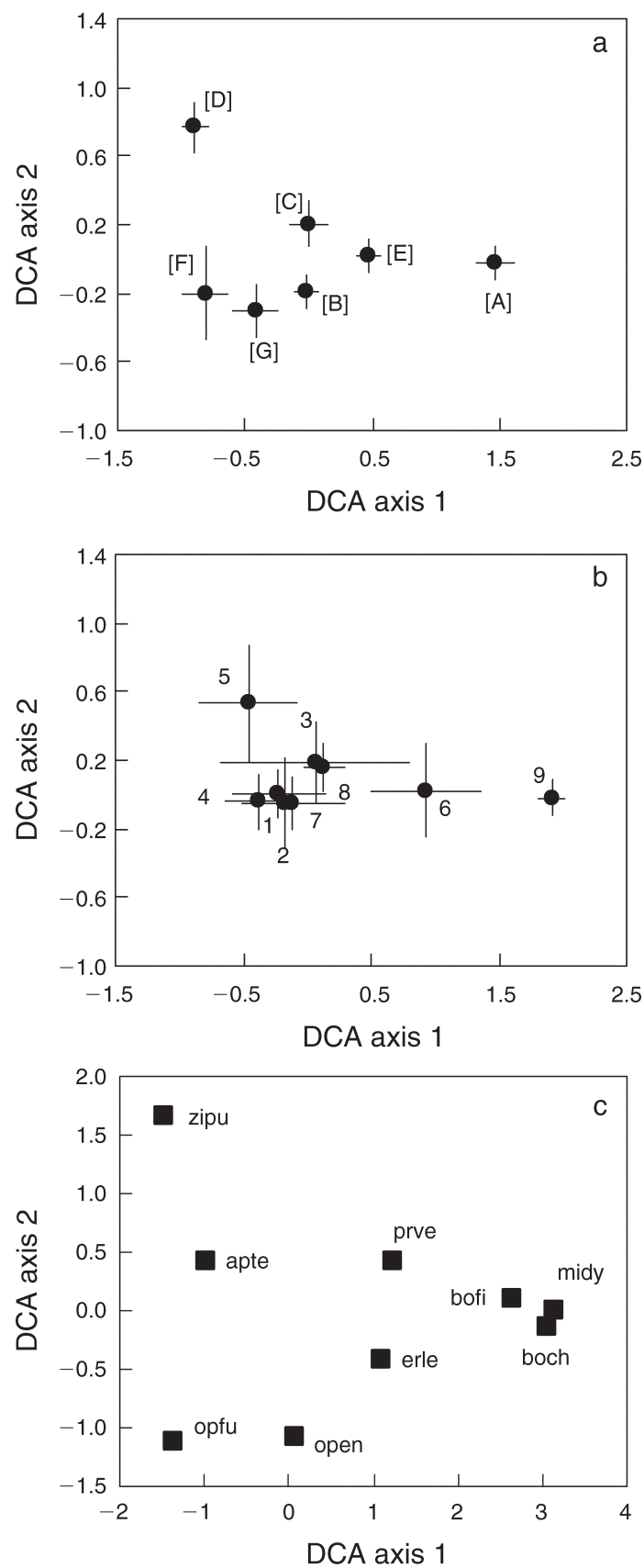

FIG. 4. (a) Mean position of seven plant communities in ordination space created by detrended correspondence analysis (DCA) on the SRER. (b) Corresponding positions of ecological site descriptions (ESDs) developed by USDA-NRCS. The three most prominent ESDs are: 1, sandy loam upland; 2, sandy loam deep; and 3, loamy upland. Minor ESDs are: 4, clay loam upland; 5 , limy upland deep; 6 , loamy slopes; 7 , sandy upland; 8 , sandy wash; and 9, granitic hills. Error bars represent \pm SD. (c) Ordination scores of key plant species in DCA. Abbreviations for species names are as in Fig. 3.

Most notably, the number of communities, or phases in the expert model, was comparable to the empirical patterns. Further, the emphasis on mesquite in the expert model appears to match its prominent role in
TABLE 1. Summary of transitions (from time $t 1$ to $t 2$ ) between seven plant communities, $[\mathrm{A}]$ though $[\mathrm{G}]$, at the Santa Rita Experimental Range, Arizona, USA.

\begin{tabular}{lccccccc}
\hline \hline & \multicolumn{7}{c}{ Community } \\
\cline { 2 - 8 } Community & {$[\mathrm{A}]_{t 2}$} & {$[\mathrm{~B}]_{t 2}$} & {$[\mathrm{C}]_{t 2}$} & {$[\mathrm{D}]_{t 2}$} & {$[\mathrm{E}]_{t 2}$} & {$[\mathrm{~F}]_{t 2}$} & {$[\mathrm{G}]_{t 2}$} \\
\hline$[\mathrm{A}]_{t 1}$ & $(90.9)$ & 1 & 3 & 0 & 7 & 0 & 1 \\
{$[\mathrm{~B}]_{t 1}$} & 1 & $(70.5)$ & 10 & 1 & 16 & 1 & 9 \\
{$[\mathrm{C}]_{t 1}$} & 3 & 19 & $(77.6)$ & 7 & 25 & 10 & 1 \\
{$[\mathrm{D}]_{t 1}$} & 0 & 2 & 4 & $(80.4)$ & 4 & 10 & 5 \\
{$[\mathrm{E}]_{t 1}$} & 2 & 17 & 8 & 1 & $(76.9)$ & 1 & 3 \\
{$[\mathrm{~F}]_{t 1}$} & 0 & 2 & 15 & 13 & 5 & $(77.4)$ & 16 \\
{$[\mathrm{G}]_{t 1}$} & 0 & 11 & 2 & 1 & 4 & 12 & $(78.0)$
\end{tabular}

Notes: Off-diagonal entries represent the number of transitions between a pair of participant communities between two consecutive samples $(t 1$ and $t 2)$. The diagonal entries, in parentheses, represent the percentage of occasions where a sample did not undergo transition, and indicate resilience. Community [A] remained unchanged most often (90.9\%), while [B] underwent frequent transitions (70.5\%).

historical dynamics, although all perennial grasses are grouped together. However, the lack of emphasis on cacti denotes a point of departure between the expert models and the empirical trends in the historical record. Also, similar to the historical dynamics, the expert

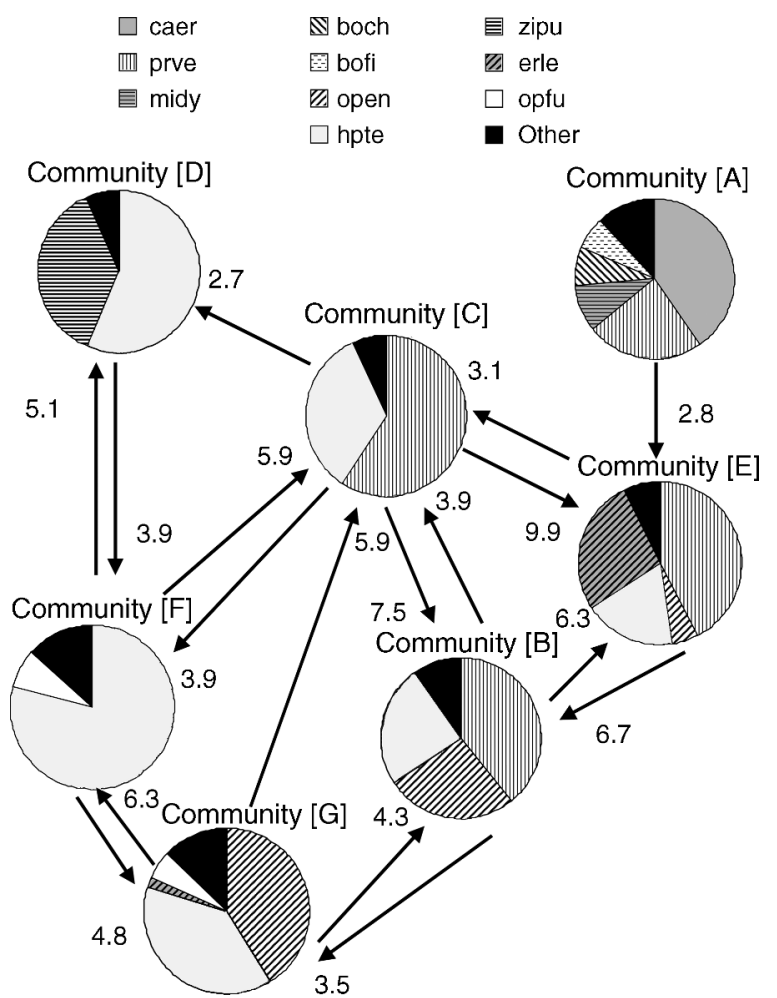

FIG. 5. Empirical patterns of transitions between seven plant communities on the Santa Rita Experimental Range (SRER) based on vegetation dynamics between 1957 and 2009. Each pie chart summarizes species composition of the communities, and arrows indicate transitions between a pair of participant communities. Numbers next to arrows represent the percentage of all transitions ( $n=253$ cases) between a pair of communities. Abbreviations for species names are as in Fig. 3. 

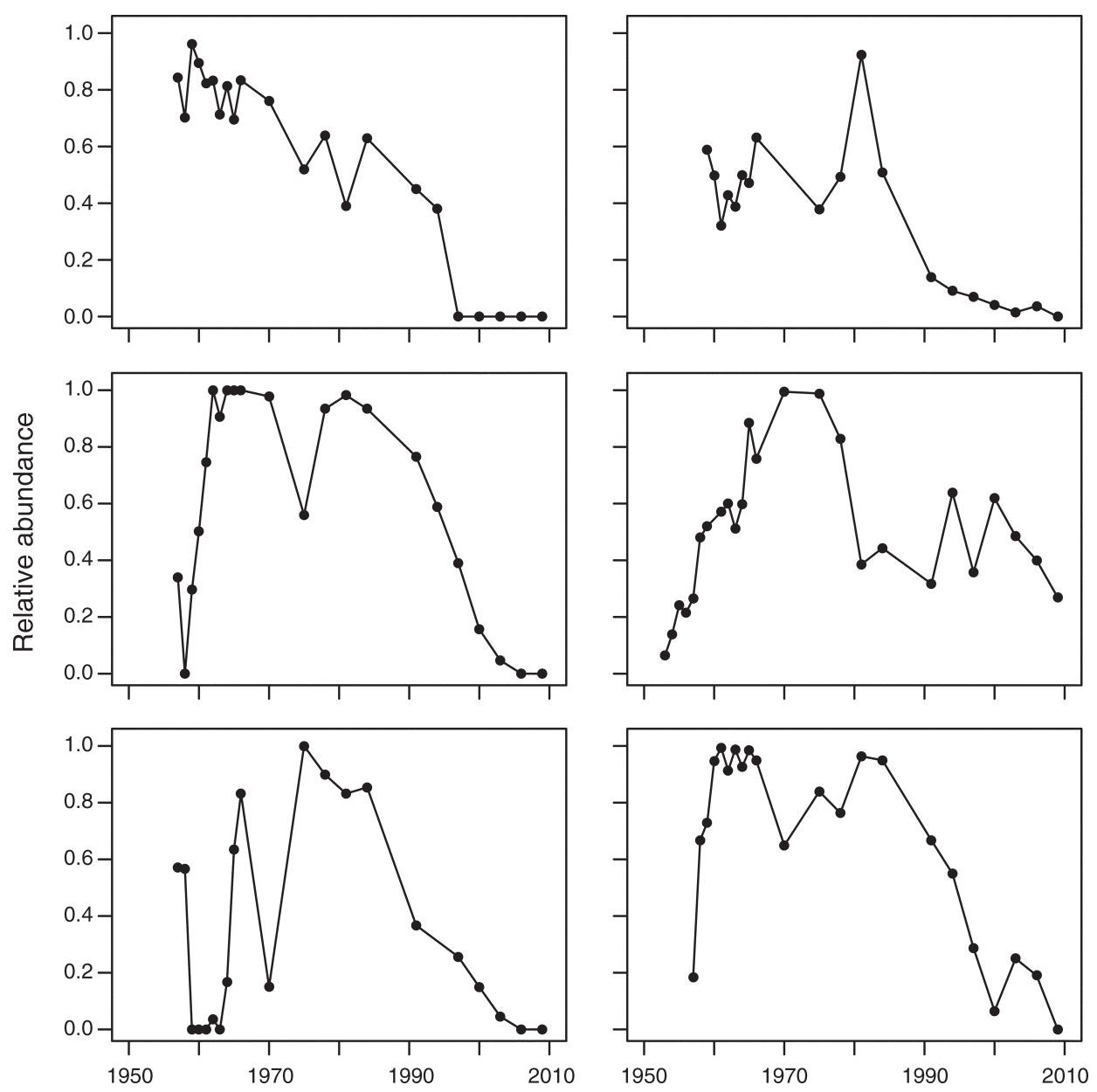

FIG. 6. Illustrative examples of transects where exceedingly large changes in species composition were detected. These transects, at least at one point in time, have been dominated by burroweed (Haplopappus tenuisecta), and have been classified as community $[\mathrm{F}]$. Each panel represents the change in relative abundance of burroweed over time in six representative transects.

model suggests that the community featuring Lehmann' lovegrass can transition into a mesquite-dominated community. But, unlike the expert model, the empirical record indicates that transitions between communities featuring the native Bouteloua grasses and Lehmann's lovegrass are likely to be rare.

\section{Trajectories of individual transects through time}

Several patterns of compositional change were identified among the various transects based on their relative position within DCA ordination-space through time (see Appendix). Illustrative examples show that species composition can remain remarkably stable for over five decades, as seen in transects within communities [G] and $[C]$, both of which were in sandy loam upland ESD (Fig. $8 \mathrm{a}, \mathrm{b})$. There were also cases of seemingly nondirectional compositional change in transects of sandy loam deep and sandy loam upland ESD (Fig. 8c, d). In addition, there were instances where initial compositional changes were reversed such that transects regained their previous composition and community designation within 10-12

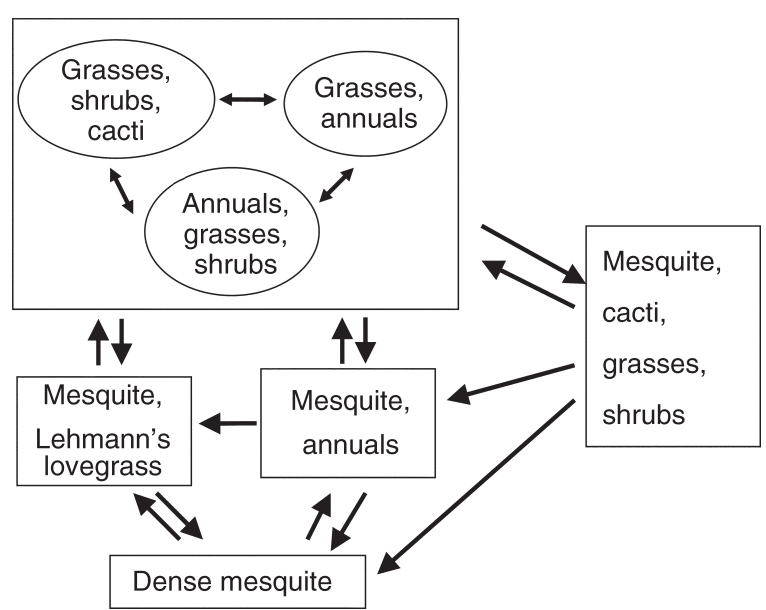

FIG. 7. The representative expert STM (state-and-transition model) for Sonoran Desert vegetation, based on NRCS description, for comparison against the empirical trends summarized in Fig. 5. The expert model recognizes eight communities (ovals), of which three are considered phases within a state (rectangle). 
years, as seen in transects in sandy loam deep and sandy loam upland ESD (Fig. 8e,f). Finally, directional changes in composition were observed where transects did not return to their previous composition. Two examples with transects in sandy loam deep ESD show that they did not return to their former compositional status within 25-36 years (Fig. 8g, h), and these may reflect irreversible shifts and the occurrence of threshold conditions. Broadly, these representative behaviors were neither exclusive to any topo-edaphic category, nor were they restricted to any particular community type. Also, nondirectional changes and reversible changes appeared to be more common than stability and directional shifts (see Appendix).

\section{Influence of precipitation}

Precipitation records over the 12 months preceding sampling showed fluctuations around the long-term average of $362 \mathrm{~mm}$ (1922-2009), with the prominent wet period in the 1980s, but prolonged drought periods did not occur (Fig. 9). The relatively drier periods received $\sim 200 \mathrm{~mm}$ of precipitation, while the wettest period received $550 \mathrm{~mm}$. The number of transitions in the vegetation record also varied through time (Fig. 9a), and was positively correlated with precipitation $(r=0.53, P=$ 0.01 ); a higher number of transitions occurred during relatively wetter periods. On average, greater species dissimilarity was expressed when frequency of transitions was high $(r=0.36, P=0.09)$, and consequently, species dissimilarity (Fig. 9b) was also seen to be positively correlated with precipitation $(r=0.35, P=$ 0.07 ).

\section{DisCUSSION}

Analysis of this 56-year vegetation record indicates that: (1) Substantial vegetation dynamics occurred in this Sonoran Desert landscape, even in the absence of management interventions; (2) an analytical protocol based on multivariate statistical tools was successful in partitioning these dynamics as transitions among seven constituent plant communities; (3) transitions due to relatively large changes in species composition were rare (18 cases out of 253); (4) distinctiveness of plant communities, as well as transitions between them, were not rigidly constrained by topo-edaphic conditions; (5) many communities and transitions were accurately described by the representative expert STM, but there were noticeable differences as well; (6) temporal dynamics in individual transects ranged from stability to directional changes that were not reversed even after two to three decades; and (7) the incidence of transitions, and accompanying species dissimilarity, were positively correlated with precipitation fluctuations.

\section{Analytical protocol}

A heuristically simple analytical protocol (based on cluster analysis and ordination) was able to express long-term vegetation dynamics as unique communities
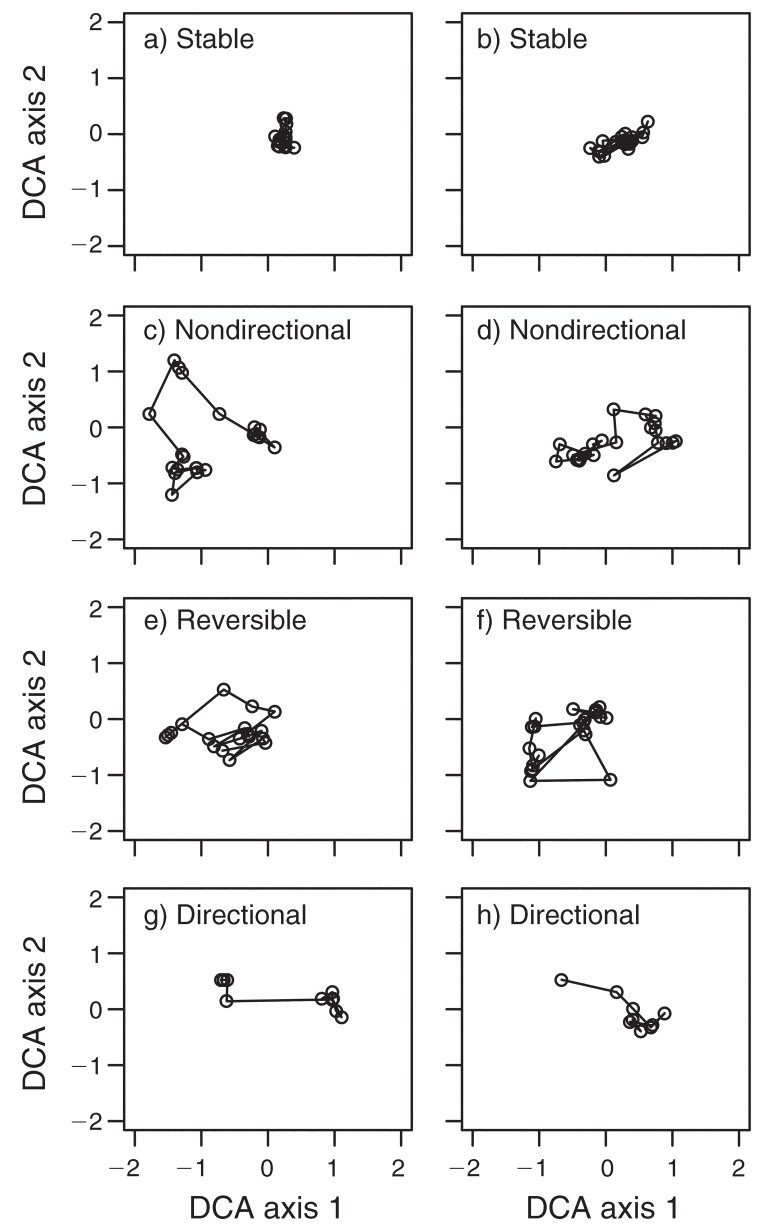

FIG. 8. Illustrative patterns of vegetation dynamics identified in the long-term vegetation record from the Santa Rita Experimental Range (SRER). Each panel represents the location of a specific transect over two-dimensional ordination space created by detrended correspondence analysis (DCA) at successive sampling periods through time. Patterns include $(\mathrm{a}, \mathrm{b})$ high stability, $(\mathrm{c}, \mathrm{d})$ nondirectional drift, $(\mathrm{e}, \mathrm{f})$ reversible change, and $(\mathrm{g}, \mathrm{h})$ directional change that was not reversed within a timescale of 20 years or more.

and transitions among them to evaluate resilience concepts. Although there is a large body of literature on resilience concepts (Mayer and Rietkerk 2004, Scheffer 2009, Briske et al. 2010), considerable uncertainties persist in recognizing their empirical signatures with statistical tools (Anderson et al. 2009), and in identifying appropriate timescales for management action (Knapp et al. 2011). Few attempts have been made to construct empirical STMs, and they have often been restricted to relatively short timescales (Allen-Diaz and Bartolome 1998, Jackson and Bartolome 2002). Our analyses could quantify community transitions, ascertain their frequency and their directionality, provide additional metrics such as the magnitude of compositional change accompanying various transitions, and also distinguish among various dynamics that occur at different time scales. This indicates that since long-term 

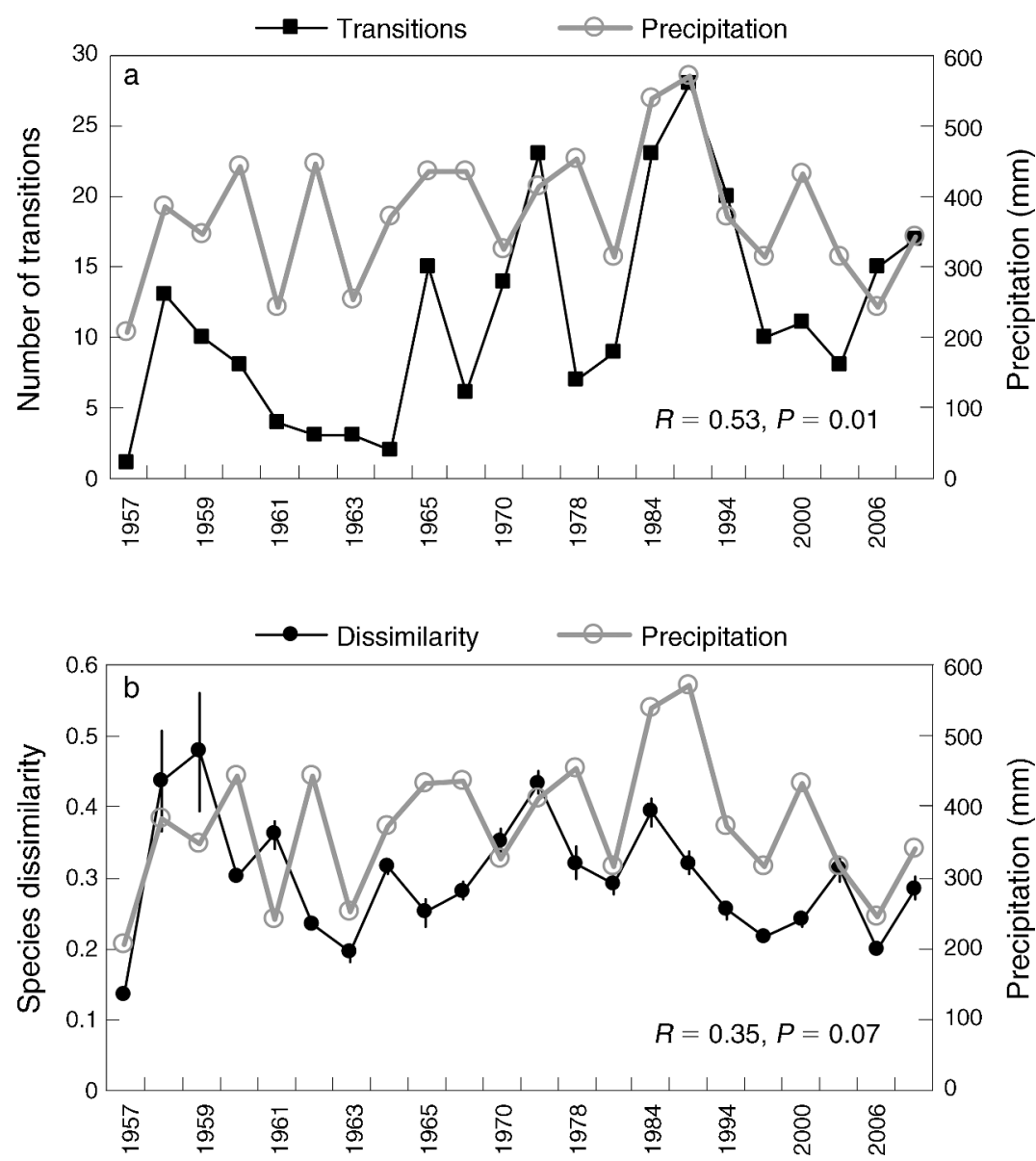

FIG. 9. (a) Mean annual precipitation recorded at six weather stations in Santa Rita Experimental Range (SRER) between 1957 and 2009, and the number of community transitions identified in the vegetation over the same period. (b) The magnitude of compositional dissimilarity accompanying these transitions and mean precipitation; error bars indicate \pm SE. Patterns indicate that a greater number of transitions occur, and greater compositional dissimilarity is expressed, during wet periods.

data can, when available, quantify potential pathways of community dynamics, they can reduce the ambiguity surrounding resilience concepts (Knapp et al. 2011), and support the construction and interpretation of STMs.

\section{Interpretation of transitions and thresholds}

The quantitative assessment of community transitions can be interpreted using resilience concepts and thereby strengthen the STM framework. While a majority of all community transitions (37 out of 42 possibilities) were realized, some were clearly more prevalent than others; they varied greatly in the magnitude of dissimilarity expressed, and some community transitions did not occur at all (Fig. 5). Community transitions in one direction were not necessarily equally prevalent in the opposite direction (Fig. 5), suggesting a disproportionate influence of potential drivers on expression of resilience among communities. Expectedly, the frequency with which transitions occurred was reflected by the compositional similarity among communities; if communities shared a dominant species, then transitions occurred more frequently. For example, community [A] composed of fairy duster and mesquite (Figs. 3 and 4), was very distinct, and was involved in very few transitions (Fig. 5, Table 1). In contrast, the communities characterized by mesquite, burroweed, and prickly pear cacti ([B], [C], and [E]; Figs. 3 and 4) were closely related and showed frequent transitions (Fig. 5, Table 1).

Similarity of community structure and species composition represents a central criterion for the development and interpretation of STMs (Briske et al. 2008), and we quantified additional characteristics such as transition frequency and the magnitude of species dissimilarity accompanying these transitions. Even though a total of 253 community transitions were recorded, only 18 were characterized by relatively large compositional shifts. Are these disproportionately large compositional shifts linked with thresholds? Alternatively, do the five types of transition pathways that did not occur in this 56-year record represent thresholds? While long-term data cannot distinguish between these 


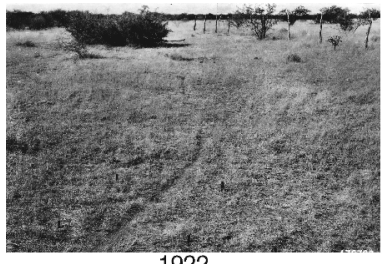

1922

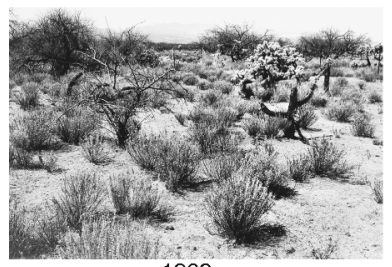

1969

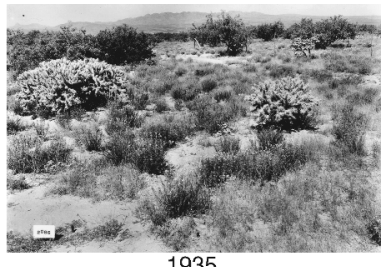

1935

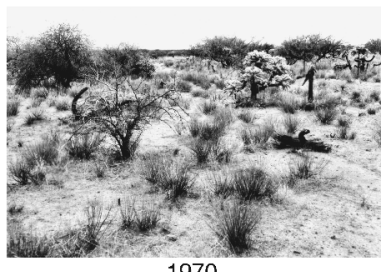

1970

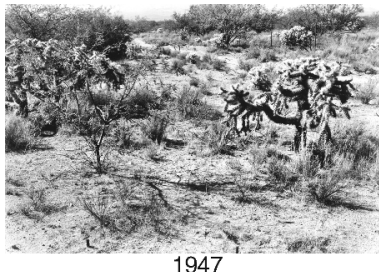

1947

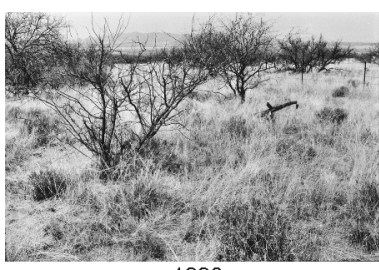

1990
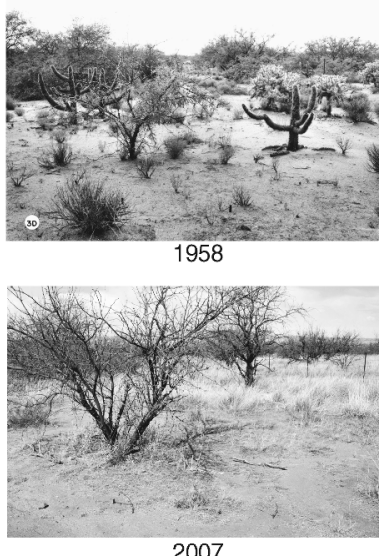

2007

Plate 1. Repeat photography (1922-2007) of vegetation changes at permanent photo station 111 on the Santa Rita Experimental Range, near Tucson, Arizona, USA. The view includes areas grazed by livestock in the foreground and an ungrazed exclosure since 1903 (Rodent Station, $1086 \mathrm{~m}$ a.s.1., $370 \mathrm{~mm}$ MAP, 19.5 $5^{\circ} \mathrm{C}$ MAT) beyond the fence in the background. In the 1922 photo, there is one large Celtis pallida (desert hackberry), scattered Prosopis velutina (mesquite), and relatively continuous herbaceous cover that was probably Bouteloua rothrockii (Rothrock grama) or annual grama species (B. aristidoides or B. barbata). In 1935, Haplopappus tenuisecta (burroweed) and Cylindropuntia fulgida (chainfruit cholla) have become established. By 1947, $H$. tenuisecta and C. fulgida have begun to decline, while $P$. velutina is becoming established on both sides of the exclosure fence. In 1958, H. tenuisecta and C. fulgida died, and the left half of the small P. velutina (foreground) died during the drought of the mid 1950s. In 1969, the third eruption since 1900 of H. tenuisecta is fully developed, but by 1970 there was a rapid decline of this species. In 1990, the nonnative grass Eragrostis lehmanniana (Lehmann lovegrass) is very abundant, and beneath that grass canopy are the H. tenuisecta established in the late 1970s. By 2007, there was a decline of H. tenuisecta and the nonnative E. lehmanniana. Images were provided by Santa Rita Experimental Range Digital Database, and more examples of repeat photography are available at: http://cals.arizona.edu/srer/photos.html

alternative interpretations, interestingly, a majority of the large transitions ( $n=14$ out of 18) involved community $[\mathrm{F}]$, featuring burrowed. This may suggest that compositional characteristics such as species identity, and associated life-history traits, could determine which communities are prone to large compositional turnover. However, burroweed is known to express large and frequent shifts in population abundance (McClaran et al. 2010), suggesting that cyclic dynamics should not be interpreted as thresholds. On the other hand, the five types of transition pathways that were not recorded all involved community [A], which represents a small fraction of the overall landscape that is closely associated with a minor ESD (granitic hills; Table 1). As such, community [A] likely represents a unique species assemblage that is restricted to relatively rare topo-edaphic conditions, rather than a threshold.

Since an overwhelming majority of community transitions in SRER appear to be inconsistent with thresholds, it may be more appropriate and informative to interpret these dynamics as community phases within a state. Land managers are more likely to confront community phase dynamics on a regular basis, than they are thresholds (Watson et al. 1996, Briske et al. 2005, Briske et al. 2008). In fact, an inordinate emphasis on thresholds may allow the vast majority of vegetation dynamics to remain unnoticed, even though these are perhaps more informative of resilience and may be more representative of signals to initiate management interventions. The varied trajectories of vegetation dynamics within individual transects, ranging from stability to directional shifts in community composition, likely comprise community phase dynamics within states (Fig. 8). When characteristics such as the frequency of particular transition pathways, their reversibility over relevant timescales, and accompanying species dissimilarity, are empirically quantified, they can support the construction and interpretation of STMs.

Empirical vegetation records may be especially useful to identify temporal scales of community phase and state dynamics that are relevant for ecosystem management (Friedel et al. 1993). For instance, two transects on the sandy loam deep ESD underwent directional changes and did not return to their former configuration even after 25-36 years (Fig. 8g, h). In both cases, burroweed was the dominant species until a series of wet years occurred during the mid-1980s, at which time Lehmann's lovegrass became dominant. Even though these changes were not reversed in two to three decades, they may represent cyclic dynamics in burroweed that are expressed over intervals of three to four decades or longer (McClaran et al. 2010). Without the insights provided by long-term vegetation records, these dynamics may remain unrecognized or may be misinterpreted.

Analysis of empirical data at the scale of individual transects provides additional insight into the occurrence of thresholds. Assuming that community transitions with large compositional dissimilarity in the burroweed community actually represented thresholds, it begs the question: Does this compositional change reflect a 
sufficient proportion of the ecological site to constitute a meaningful threshold that necessitates a management response? Thresholds are often assumed to involve compositional changes that encompass a large proportion, or even the entire ecological site under consideration. Therefore, in addition to characteristics such as species dissimilarity and reversibility, spatial characteristics are also an important consideration in designating thresholds. Our analysis provides additional insights into the complexities of thresholds, and illustrates the practical challenges for their interpretation within the STM framework.

\section{Climatic influence}

The greater incidence of community transitions during periods of relatively high precipitation illustrates the importance of climatic drivers in vegetation dynamics (Fig. 9a). Furthermore, species dissimilarities accompanying these transitions were also greater during relatively wet periods (Fig. 9b). These correlations suggest that even coarse climatic projections, such as El Niño and La Niña phenomena, which concern broadly predictable changes in precipitation, may be useful for site-specific ecosystem management (Swetnam et al. 1999). These vegetation dynamics are likely of autogenic origin because intensive management practices had not been applied to these transects and livestock grazing regimes have also remained consistent throughout this period (McClaran et al. 2003, Mashiri et al. 2008). This emphasizes that climatic variation and its interaction with autogenic mechanisms of vegetation dynamics merit greater attention in the STM framework.

A potential explanation for the influence of precipitation on species composition in this semiarid ecosystem is that community responses are often linked to resource-mediated fluctuations in the relative strengths of intraspecific and interspecific competition (Chesson et al. 2004). High precipitation is expected to intensify intraspecific competition relative to interspecific competition (Chesson et al. 2004), and these mechanisms may relate to the large number of transitions associated with periods of high precipitation. However, these patterns should not be automatically extrapolated into a corollary effect of droughts, since these data (19532009) immediately followed, but did not encompass a significant drought period (Fig. 9).

\section{Correspondence with expert models}

How well do the community transitions observed in this long-term vegetation record correspond with the community phases and states, and associated dynamics expressed in expert models? STMs are expected to encompass the full range of potential vegetation dynamics, although certain transition pathways can be selectively eliminated by appropriate management interventions. The generalized expert model effectively represents the temporal dynamics of important species such as mesquite, but does not capture the increasing abundance of cacti during the last two decades. In general, we documented relatively short periods of stability in this Sonoran Desert ecosystem that were frequently punctuated by substantial, but often reversible, vegetation change.

Our analysis was able to identify specific communities, the frequency, and temporal scales of potential transition pathways among them. We observed that community transitions were influenced by climatic drivers, perhaps in concert with autogenic processes, and that these dynamics were only weakly constrained by soil types and topographic features. Metrics derived from these community transitions, including transition frequency, magnitude of accompanying compositional change, presence of unidirectional trajectories, and lack of reversibility within various timescales, can inform the construction and interpretation of STMs. Empirical vegetation records can also provide valuable insights for interpreting ambiguous resilience concepts to more effectively guide ecosystem management.

\section{ACKNOWLEDGMENTS}

We are grateful to all researchers who established and monitored the vegetation transects to create the long-term database. Data were provided by the Santa Rita Experimental Range Digital Database, with support from USDA Forest Service Rocky Mountain Research Station and the University of Arizona. Our research was supported in part by USDA CSREES grant 2007-38415-18637. Critiques by David J. Augustine and an anonymous referee helped improve an earlier draft.

\section{Literature Cited}

Allen-Diaz, B., and J. W. Bartolome. 1998. Sagebrush-grass vegetation dynamics: Comparing classical and state-transition models. Ecological Applications 8:795-804.

Anderson, T., J. Carstensen, E. Hernández-García, and C. M. Duarte. 2009. Ecological thresholds and regime shifts: approaches to identification. Trends in Ecology and Evolution 24:49-57.

Archer, S., C. Scifres, C. R. Bassham, and R. Maggio. 1988. Autogenic succession in a subtropical savanna: Conversion of grasslands to thorn woodland. Ecological Monographs 58:111-127.

Bestelmeyer, B. T., J. E. Herrick, J. R. Brown, D. A. Trujillo, and K. M. Havstad. 2004. Land management in the American Southwest: a state-and-transition approach to ecosystem complexity. Environmental Management 34:3851.

Breckenfield, D. J., and D. Robinett. 2003. Soil and ecological sites of the Santa Rita Experimental Range. Pages 157-165 in M. P. McClaran, P. F. Ffolliot, and C. B. Edminister, editors. Santa Rita Experimental Range: 100 years (19032003). USDA Forest Service, Rocky Mountain Research Station, Ogden, Utah.

Briske, D. D., B. T. Bestelmeyer, T. K. Stringham, and P. L. Shaver. 2008. Recommendations for development of resilience-based state-and-transition models. Rangeland Ecology and Management 61:359-367.

Briske, D. D., S. D. Fuhlendorf, and F. E. Smeins. 2003. Vegetation dynamics on rangelands: a critique of the current paradigms. Journal of Applied Ecology 40:601-614.

Briske, D. D., S. D. Fuhlendorf, and F. E. Smiens. 2005. Stateand-transition models, thresholds, and rangeland health: A 
synthesis of ecological concepts and perspectives. Rangeland Ecology and Management 58:1-10.

Briske, D. D., R. A. Washington-Allen, C. R. Johnson, J. A. Lockwood, D. R. Lockwood, T. K. Stringham, and H. H. Shugart. 2010. Catastrophic thresholds: A synthesis of concepts, perspectives, and applications. Ecology and Society 15:37.

Chesson, P., R. L. E. Gebauer, S. Schwinning, N. Huntly, K. Wiegand, M. S. K. Ernest, A. Sher, A. Novoplansky, and J. F. Weltzin. 2004. Resource pulses, species interactions, and diversity maintenance in arid and semi-arid environments. Oecologia 141:236-253.

Cumming, G. S., G. Barnes, S. Perz, M. Schmink, K. E. Sieving, J. Southworth, M. Binford, R. D. Holt, C. Stickler, and T. Van Holt. 2005. An exploratory framework for the empirical measurement of resilience. Ecosystems 8:975-987.

Dyksterhuis, E. J. 1949. Condition and management of rangeland based on quantitative ecology. Journal of Range Management 2:104-115.

Fraley, C., and A. E. Raftery. 2002. Model-based clustering, discriminant analysis, and density estimation. Journal of the American Statistical Association 97:611-632.

Friedel, M. H., G. Pickup, and D. J. Nelson. 1993. The interpretation of vegetation change in a spatially and temporally diverse arid Australian landscape. Journal of Arid Environments 24:241-260.

Gillson, L., and M. T. Hoffman. 2007. Rangeland ecology in a changing world. Science 315:53-54.

Gunderson, L. H. 2000. Ecological resilience: in theory and application. Annual Review of Ecology and Systematics 31:425-439.

Hastings, A., and D. B. Wysham. 2010. Regime shifts in ecological systems can occur with no warning. Ecology Letters 13:464-472.

Hill, M. O., and H. G. Gauch, Jr. 1980. Detrended correspondense analysis: an improved ordination technique. Vegetatio 42:47-58.

Holling, C. S. 1973. Resilience and stability of ecological systems. Annual Review of Ecology and Systematics 4:1-23.

Jackson, R. D., and J. W. Bartolome. 2002. A state-andtransition approach to understanding nonequilibrium plant community dynamics in Californian grasslands. Plant Ecology 162:49-65.

King, E. G., and R. J. Hobbs. 2006. Identifying linkages among conceptual models of ecosystem degradation and restoration: towards an integrative framework. Restoration Ecology 14:369-378.

Knapp, C. N., and M. E. Fernandez-Gimenez. 2009. Understanding change: Integrating rancher knowledge into stateand-transition models. Rangeland Ecology and Management 62:510-521.

Knapp, C. N., M. E. Fernandez-Gimenez, D. D. Briske, B. T. Bestelmeyer, and X. B. Wu. 2011. An assessment of stateand-transition models: Perceptions following two decades of development and implementation. Rangeland Ecology and Management 64:598-606.

Legendre, L., and P. Legendre. 1998. Numerical ecology. Second edition. Elsevier Science, Amsterdam, The Netherlands.
Mashiri, F. E., M. P. McClaran, and J. S. Fehmi. 2008. Short- and long-term vegetation change related to grazing systems, precipitation, and mesquite cover. Rangeland Ecology and Management 61:368-379.

Mayer, A. L., and M. Rietkerk. 2004. The dynamic regime concept for ecosystem management and restoration. BioScience 54:1013-1020.

McClaran, M. P., and D. L. Angell. 2007. Mesquite and grass relationships at two spatial scales. Plant Ecology 191:119 126.

McClaran, M. P., D. M. Browning, and C. Huang. 2010. Temporal dynamics and spatial variability in desert grassland vegetation. Pages 145-166 in R. H. Webb, D. E. Boyer, and R. M. Turner, editors. Repeat photography: Methods and application in natural sciences. Island Press, Washington, D.C., USA.

McClaran, M. P., P. F. Folliott, and C. B. Edminster. 2003. Santa Rita Experimental Range: 100 years (1903 to 2003) of accomplishments and contributions. USDA Forest Service, Rocky Mountain Research Station, Fort Collins, Colorado USA.

Milligan, G. W., and M. C. Cooper. 1985. An examination of procedures for determining the number of clusters in a data set. Psychometrika 50:159-179.

R Development Core Team. 2008. R: a language and environment for statistical computing. R Foundation for Statistical Computing, Vienna, Austria. http://www. R-project.org.

Reynolds, J. F., et al. 2007. Global desertification: Building a science for dryland development. Science 316:847.

Scheffer, M. 2009. Critical transitions in nature and society. Princeton University Press, Princeton, New Jersey, USA.

Scheffer, M., J. Bascompte, W. A. Brock, V. Brovkin, S. R. Carpenter, V. Dakos, H. Held, E. H. Van Nes, M. Rietkerk, and G. Sugihara. 2009. Early-warning signals for critical transitions. Nature 461:53-59.

Scheffer, M., S. Carpenter, J. A. Foley, C. Folke, and B. Walker. 2001. Catastrophic shifts in ecosystems. Nature 413:591-596.

Scheffer, M., S. P. Hosper, M. L. Meijer, and B. Moss. 1993. Alternative equilibria in shallow lakes. Trends in Ecology and Evolution 8:275-279.

Stringham, T. K., W. C. Krueger, and P. L. Shaver. 2003. State and transition modeling: an ecological process approach. Journal of Range Management 56:106-113.

Swetnam, T. W., C. D. Allen, and J. L. Betancourt. 1999. Applied historical ecology: using the past to manage for the future. Ecological Applications 9:1189-1206.

Walker, S., and J. B. Wilson. 2002. Tests for nonequilibrium, instability, and stabilizing processes in semiarid plant communities. Ecology 83:809-822.

Watson, I. W., D. G. Burnside, and A. M. Holm. 1996. Eventdriven or continuous: Which is the better model for managers? Rangeland Journal 18:351-369.

Westoby, M., B. H. Walker, and I. Noy-Meir. 1989. Opportunistic management for rangelands not at equilibrium. Journal of Range Management 42:266-274.

\section{Supplemental Material}

\section{Appendix}

Supplementary figures showing trajectories of individual transects at Santa Rita Experimental Range through time over ordination space (Ecological Archives A022-026-A1). 